\title{
comportement mécanique des roches en fonction de la température
}

\author{
R. HOUPERT
}

\section{F. HOMAND-ETIENNE}

\author{
Assistant \\ Centre de Recherches en Mécanique et Hydraulique des Sols et des Roches \\ Institut National Polytechnique de Lorraine - E.N.S.G., Nancy*
}

\section{INTRODUCTION}

Les recherches effectuées sur l'évolution du comportement des matériaux rocheux avec la température, à des pressions de confinement faibles ( 0 à $20 \mathrm{MPa}$ ) sont peu nombreuses. Par contre, beaucoup d'études se situent dans le domaine des hautes températures et des hautes pressions de confinement et elles ont été réalisées en vue de la compréhension de certains processus géologiques. Les projets de stockage souterrain ou de gazélfication in situ ne concernent que les faibles pressions de confinement. En compression simple, on maximise les effets de la température, puisque d'une façon générale la pression de confinement tend à atténuer les effets des hétérogénéités.

Des essais de compression simple ont été effectués dans le domaine des basses températures (jusqu'à - $196^{\circ} \mathrm{C}$ ) par KUMAR (1968), BRIGHENTI (1970), HOUPERT (1970). Toutes ces études mettent en évidence une forte augmentation de la résistance

(*) Rue du Doyen-Marcel-Roubaut, BP 40 - 54501 Vandoeuvre-lès-Nancy. ultime lorsque la température décroît. Permi les études réalisées sous faible confinement et à des températures élevées, Wingquist (1969) a suivi l'évolution des constantes élastiques depuis $27^{\circ} \mathrm{C}$ jusqu'à $827^{\circ} \mathrm{C}$. Les résultats, obtenus sur un basalte, indiquent qu'une augmentation de température diminue le module d'Young et le coefficient de Poisson. Une étude de STEPHANSSON et al. (1978) entre $20^{\circ} \mathrm{C}$ et $200^{\circ} \mathrm{C}$ sur le granite de Stripa aboutit aux mêmes conclusions. Des études concernant, en particulier, l'influence de la température sur la courbe complète contraintedéformation, ont été effectuées par HOUPERT et HOMAND-ETIENNE (1979) sur un granite et un marbre. Les constatations expérimentales montrent que le module de déformation, la limite élastique et la résistance ultime diminuent, mais que la part des déformations non élastiques augmente en fonction de la température.

Le rôle de la température dans le comportement mécanique des roches est fonction de leur nature minéralogique et de leur structure (texture et discontinuités). Les variations de température peuvent affecter la composition minéralogique (départ de certains constituants) et le réseau cristallin des grains de la roche. Les changements de structure sont principale- 
ment liés à la modification des discontinuités (fissures et pores). Dans les matériaux formés de cristaux à forte anisotropie de dilatation thermique (calcite dans le cas du marbre, quartz et feldspath dans celui des granites) ou de cristaux de dimensions ou de nature minéralogique différentes (cas des roches à texture hétérogène, comme les granites), une augmentation de température produit une variation différentielle des dimensions et du volume des minéraux, ainsi que des défauts dans les cristaux. Ces dilatations différentielles des grains de la roche donnent lieu à la formation de microfissures et l'intensité de cette microfissuration augmente avec la température. Tant que la roche est maintenue à la température sous laquelle se sont formées ces fissures, celles-ci resteront fermées. Une diminution de la température conduira à leur ouverture.

Dans l'ensemble, le comportement mécanique des roches en fonction de la température est similaire à celui des autres matériaux. La résistance ultime ou la limite de plasticité décroît avec une augmentation de la température. Par ailleurs, une augmentation de la vitesse de sollicitation mécanique conduit à une augmentation de la résistance ultime ou de la limite de plasticité. Cette connexion entre les variations de température, d'une part, et de vitesse de sollicitation, d'autre part, est suggérée par l'équation d'Arrhénius qui relie la vitesse de certains phénomènes (diffusion des atomes, variation des caractéristiques mécaniques) à la température (RICHARDS, 1965)

$$
\text { Vitesse }=\mathrm{A} \exp (-\mathrm{Q} / \mathrm{RT}) \text {, }
$$

relation dans laquelle $\mathrm{Q}$ désigne l'énergie d'activation par mole de substance, $\mathrm{R}$ la constante universelle des gaz, $\mathrm{T}$ la température absolue et $\mathrm{A}$ une constante. Cette équation s'applique directement à la déformation plastique des métaux, mais peut être utilisée pour décrire les tendances d'évolution de ces phénomènes pour les matériaux rocheux.

Dans les roches, la variation de résistance ultime en fonction de la vitesse de sollicitation repose en grande partie sur la relaxation des contraintes dans le matériau (HOUPERT, 1974). Par conséquent, cette relaxation intervient également dans la variation de la résistance ultime en fonction de la température.

Le présent travail consiste en une expérimentation générale en compression simple sur le comportement mécanique des roches en fonction de la température; elle comprend, en particulier, l'étude de l'influence de la température sur la phase post-maximum et sur le temps de relaxation en différents points de la courbe contrainte-déformation. En outre, des résultats concernant le rôle de la température dans la variation de la résistance en compression en fonction de la vitesse de sollicitation sont également présentés.

\section{ROCHES ÉTUDIÉES}

Les essais mécaniques ont porté sur les granites de Senones et de La Clarté, le marbre de Carrare, le calcaire d'Euville et le grès des Vosges.

Le granite de Senones présente une structure massive quasi isotrope; il contient du quartz en petits grains $(20 \%)$, des feldspaths $(62 \%)$, le feldspath potassique pouvant atteindre $15 \mathrm{~mm}$. Le mica $(10 \%)$ et l'amphibole $(7 \%)$ entrent également dans la composition de la roche.

Le granite de La Clarté est également à structure massive. Le quartz ( $28 \%$ ) est souvent en amas dont la taille peut atteindre $15 \mathrm{~mm}$ et, de plus, il est très fissuré. Les feldspaths constituent $65 \%$ de la roche. Le feldspath potassique est de taille importante (jusqu'à $25 \mathrm{~mm}$ ). Il existe environ $6 \%$ de mica.

Le marbre de Carrare utilisé dans le cadre de cette étude est formé de cristaux de calcite de 0,1 à $0,6 \mathrm{~mm}$. La structure paraît homogène et isotrope.

Le calcaire d'Euville est un calcaire biodétritique dans lequel prédominent les débris d'entroques (de 2 à $4 \mathrm{~mm}$ ). La phase de liaison, peu développée, est constituée de cristaux de calcite.

Le grès des Vosges est formé de grains de quartz $(40 \%)$, de feldspaths (15\%), de fragments lithiques $(30 \%)$ et d'environ $5 \%$ de petits micas. La cimentation est très peu développée et ne dépasse pas $10 \%$; par conséquent, la porosité intergranulaire est très forte. La taille des grains varie entre 0,04 et $0,05 \mathrm{~mm}$.

Ces roches diffèrent par leur porosité comme l'indique le tableau 1. Le marbre de Carrare a une porosité quasi nulle, sa perméabilité intrinsèque est également très faible. Les deux granites ont une porosité légèrement différente en valeur absolue mais qui prend une certaine importance puisqu'il s'agit d'une porosité de fissure. Le granite de La Clarté est plus fissuré que celui de Senones, ce qui se traduit, pour le premier,

Tableau I. - Principales caractéristiques physiques des roches testées

\begin{tabular}{|l|c|c|c|c|}
\hline & $\begin{array}{c}\text { Poids } \\
\text { volumique } \\
\left(\mathrm{kN} / \mathrm{m}^{3}\right)\end{array}$ & $\begin{array}{c}\text { Porosité } \\
\text { totale } \\
(\%)\end{array}$ & $\begin{array}{c}\text { Célérité } \\
\text { des ondes } \\
(\mathrm{m} / \mathrm{s})\end{array}$ & $\begin{array}{c}\text { Perméabilité } \\
\text { intrinsèque } \\
\left(\mathrm{cm}^{2}\right)\end{array}$ \\
\hline Granite de Senones........... & 26,68 & 0,3 & 4830 & $7.10^{-16}$ \\
Granite de La Clarté.......... & 26,05 & 0,5 & 3428 & $1.10^{-15}$ \\
Calcaire d'Euville............... & 22,68 & 16 & 3140 & $2.10^{-9}$ \\
Marbre de Carrare........... & 27,04 & $<0,1$ & 3817 & $10^{-16}$ \\
Grès des Vosges............... & 20,61 & 22 & 1904 & $8.10^{-10}$ \\
\hline
\end{tabular}


par une célérité des ondes plus faible et une perméabilité intrinsèque plus forte par rapport au second. Le calcaire et le grès ont des porosités élevées.

\section{ESSAIS DE COMPRESSION SIMPLE SOUS TEMPÉRATURE}

Des éprouvettes cylindriques de $5 \mathrm{~cm}$ de diamètre et d'élancement 2 ont été portées jusqu'à la température voulue suivant une vitesse d'augmentation de température de $50^{\circ} \mathrm{C} / \mathrm{h}$ jusqu'à $300^{\circ} \mathrm{C}$ et de $100^{\circ} \mathrm{C} / \mathrm{h}$ pour les températures supérieures. Des essais préliminaires (ABEGA EBOLO, 1978) ont montré que dans le domaine des faibles variations de température $\left(50^{\circ} \mathrm{C} / \mathrm{h}\right.$ à $150^{\circ} \mathrm{C} / \mathrm{h}$ ), la valeur de la vitesse de mise en température n'a pas d'influence appréciable sur le degré de fissuration de l'éprouvette. Celui-ci est évalué par la mesure de la célérité des ondes et par la résistance à la compression d'éprouvettes après retour à la température ambiante. Par contre, on constate une influence de la durée de maintien à la température maximale, qui se traduit par une diminution de la

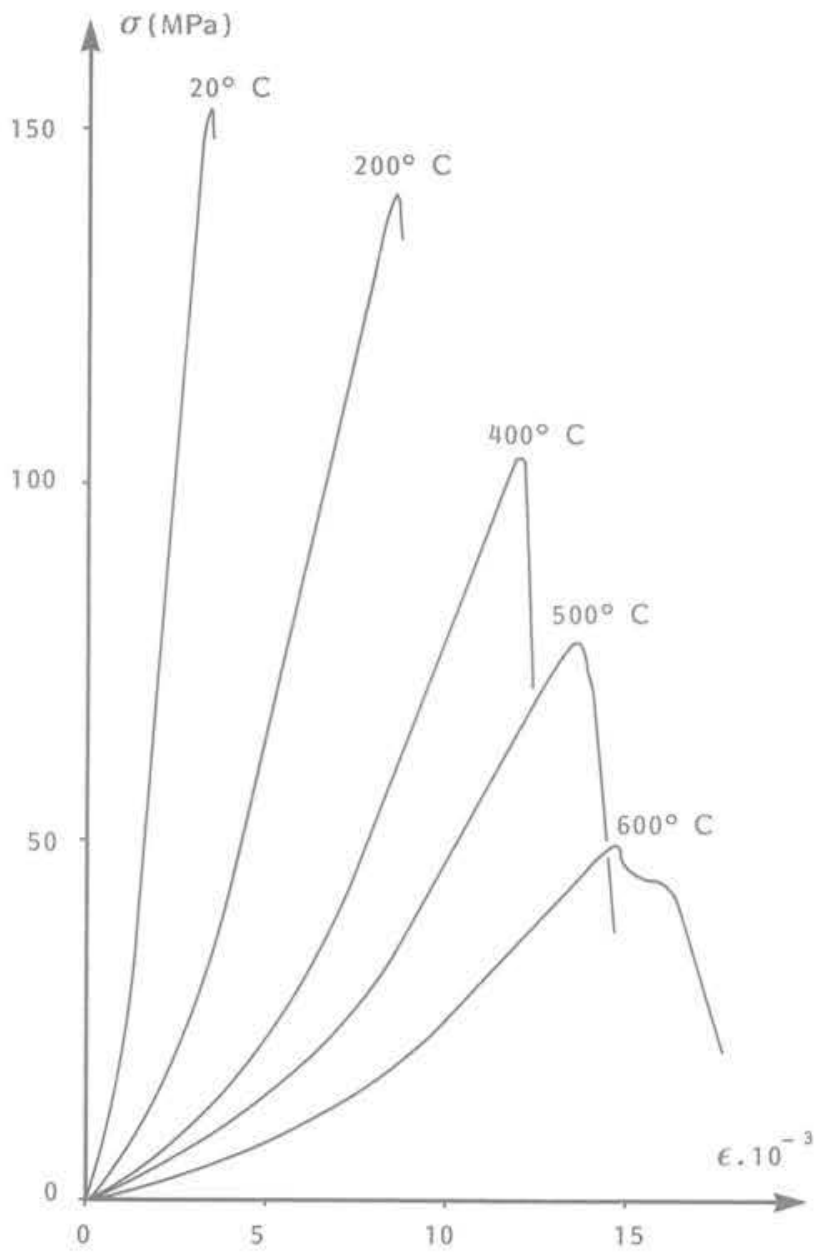

Fig. 1. - Courbes contrainte-déformation du granite de Senones à différentes températures $\left(\dot{\varepsilon}=10^{-5} s^{-1}\right)$. célérité des ondes de quelques pour cents, lorsque cette durée passe de $5 \mathrm{~h}$ à $24 \mathrm{~h}$. La durée de maintien à la température maximale a donc été fixée, pour toutes les éprouvettes, à $15 \mathrm{~h}$. Les températures d'essai ont été les suivantes: $200^{\circ} \mathrm{C}, 400^{\circ} \mathrm{C}, 500^{\circ} \mathrm{C}$ et $600^{\circ} \mathrm{C}$.

Les figures 1 à 5 montrent l'évolution de l'allure des courbes contrainte-déformation axiale en fonction de la température pour les deux granites, le calcaire, le marbre et le grès. Ces courbes ont été obtenues à l'aide d'une machine d'essai asservie et pour la vitesse de déformation $\dot{\varepsilon}=10^{-5} \mathrm{~s}^{-1}$. On remarque, sauf pour le grès, une diminution de la résistance à la compression avec l'augmentation de la température. Les courbes représentées ne sont qu'un exemple parmi les trois à cinq essais réalisés pour chaque température.

Les courbes $\sigma-\varepsilon$ du granite de Senones (fig. 1) et du granite de La Clarté (fig. 2) présentent à peu près la même allure. Les résistances à la compression des deux granites sont à peu près identiques à la température ordinaire. L'évolution de la résistance ultime en fonction de la température est nettement différente pour les deux granites. Lorsque l'on passe de $20^{\circ} \mathrm{C}$ à $200^{\circ} \mathrm{C}$, la perte de résistance du granite de La Clarté

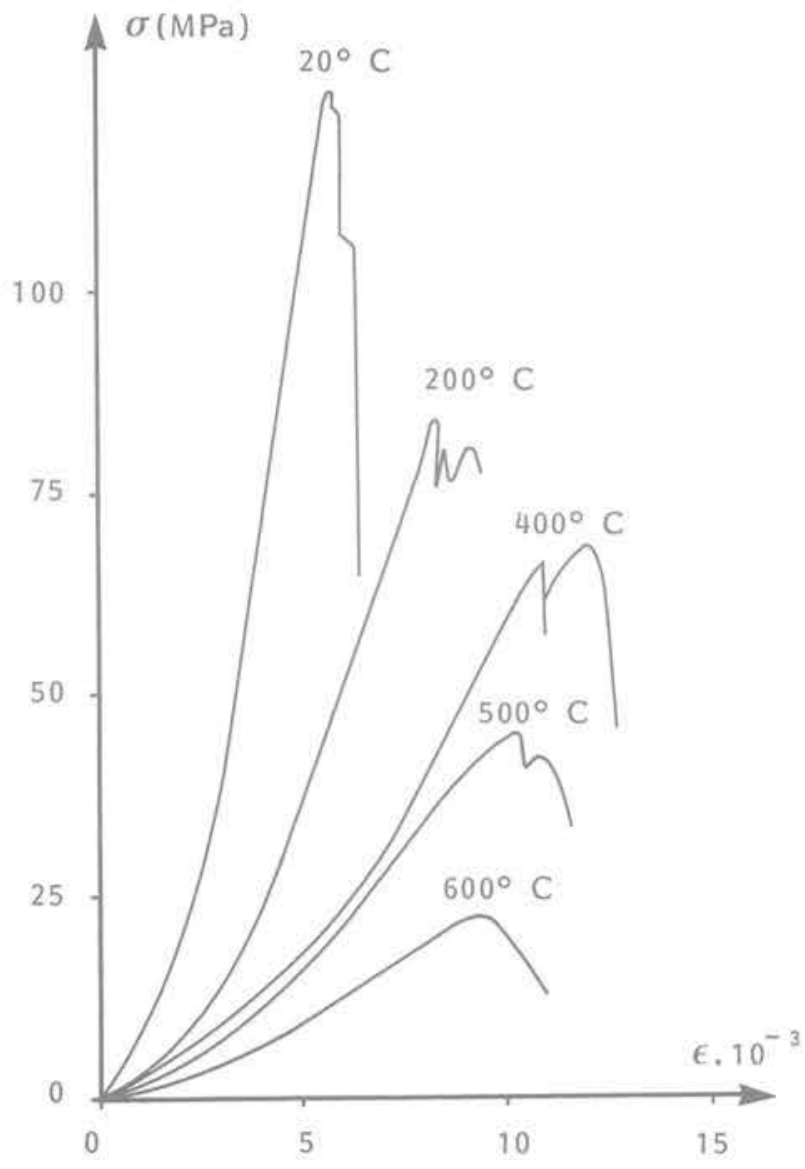

Fig. 2. - Courbes contrainte-déformation du granite de La Clarté à différentes températures $\left(\dot{\varepsilon}=10^{-5} s^{-1}\right)$. 
est de l'ordre de $45 \%$, tandis que celle du granite de Senones est d'environ $8 \%$. Pour des températures supérieures, la perte de résistance du granite de La Clarté continue à être plus importante que celle du granite de Senones. A $600^{\circ} \mathrm{C}$, elle est de $85 \%$ pour le premier et de $70 \%$ pour le second. Les modules de déformation diminuent avec la température. On remarque également un abaissement de la limite élastique, par rapport à la résistance ultime, avec l'augmentation de la température. En ce qui concerne le comportement radoucissant, on observe, d'une façon générale, que la pente post-maximum, caractérisée par le rapport de la déformation axiale totale sur la déformation axiale relative à la charge maximale, augmente avec la température; elle varie de 1 à 1,4 en passant de $20^{\circ} \mathrm{C}$ à $600^{\circ} \mathrm{C}$.

La différence de comportement entre les deux granites peut s'expliquer par une légère différence de structure. Les grains du granite de La Clarté sont plus gros que ceux du granite de Senones, en particulier, le quartz se présente en gros amas. Les différences de dilatation thermique sont plus élevées dans le granite de La Clarté, ce qui donne lieu à des fissures plus importantes dans ce granite que dans le granite de Senones.

Les courbes $\sigma-\varepsilon$ du marbre de Carrare (fig. 3) montrent une perte importante de la résistance ultime relative à $200^{\circ} \mathrm{C}(25 \%)$. Il faut cependant remarquer que la perte de résistance à $600^{\circ} \mathrm{C}$ est moins

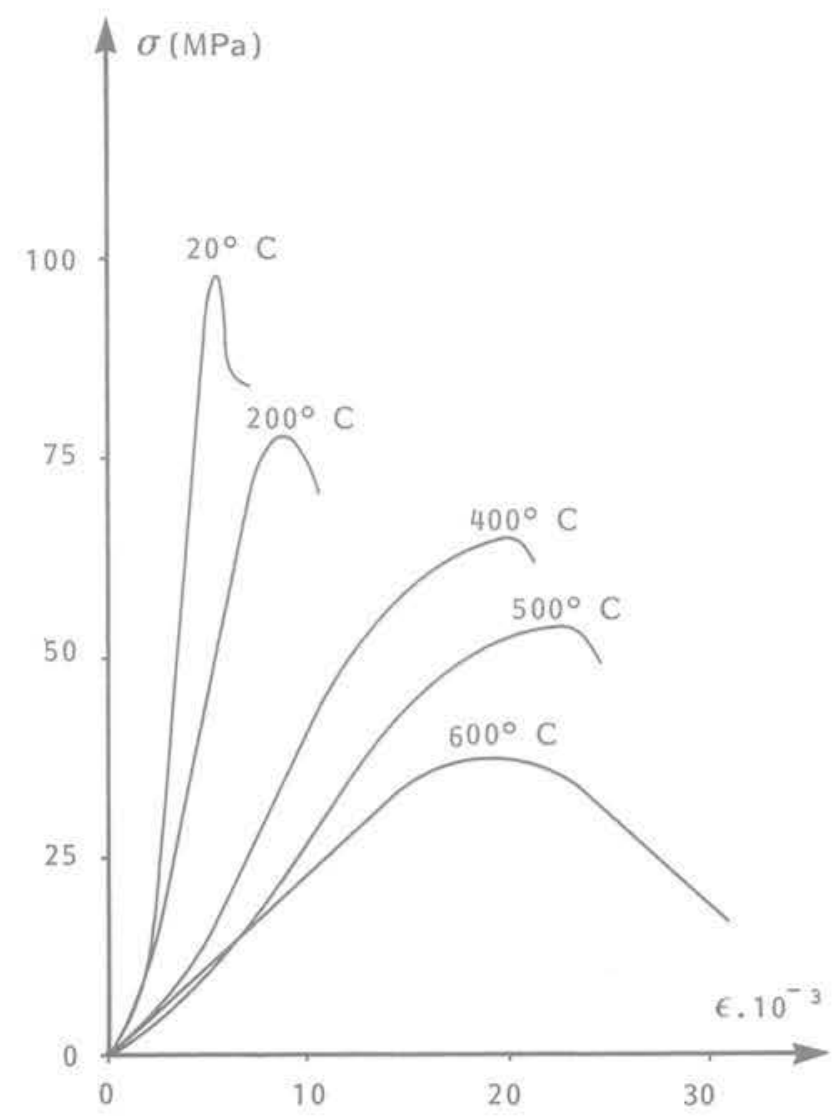

Fig. 3. - Courbes contrainte-déformation du marbre de Carrare à différentes températures $\left(\dot{\varepsilon}=10^{-5} s^{-1}\right)$, importante que dans le cas des granites. La limite élastique diminue plus fortement avec la température que dans le cas des granites. La pente post-maximum augmente de 1,2 à 1,9 avec la température. Cependant, le contrôle de la rupture n'est souvent que partiel.

Le calcaire d'Euville (fig. 4) présente une perte de résistance à la compression de l'ordre de $25 \%$ à $200^{\circ} \mathrm{C}$. Cette résistance diminue ensuite lentement jusqu'à $600^{\circ} \mathrm{C}$ (perte ue $55 \%$ ). La limite élastique est fortement réduite, tandis que la pente post-maximum augmente de 1,1 à 2,5 .

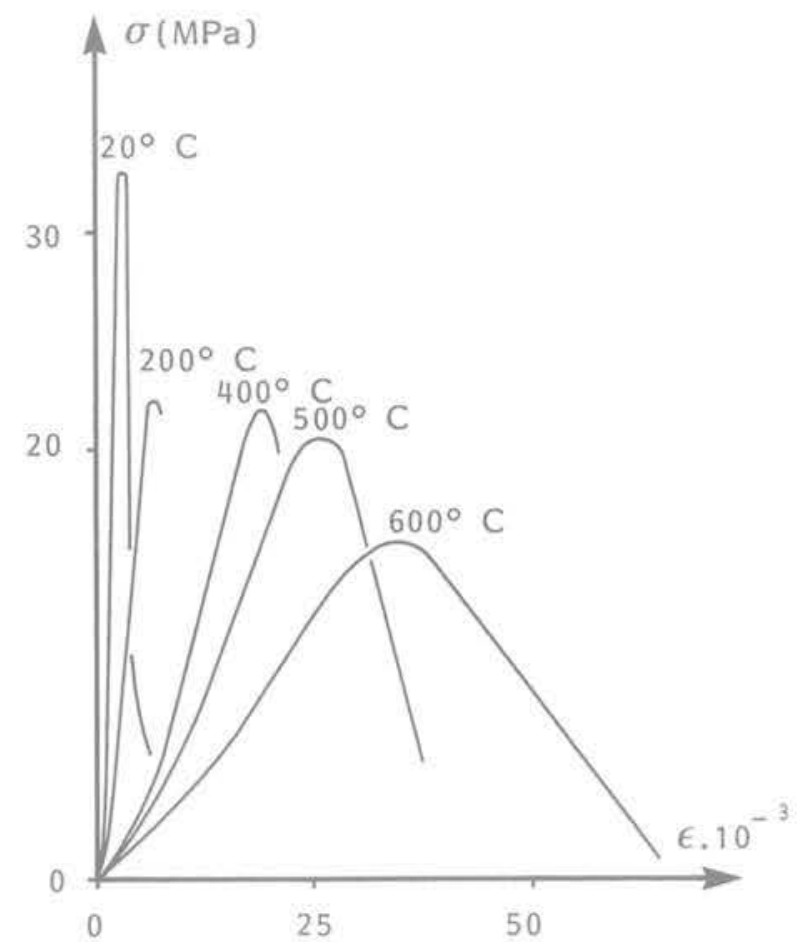

Fig. 4. - Courbes contrainte-déformation du calcaire d'Euville à différentes températures $\left(\dot{\varepsilon}=10^{-5} s^{-1}\right)$.

Le comportement du grès des Vosges (fig. 5) est complètement différent de celui des autres roches, dans ce sens que la résistance ultime ne diminue pas régulièrement avec la température; elle a plutôt tendance à augmenter pour certaines températures, bien que la dispersion des résistances ne permette pas d'apprécier avec certitude cette évolution. Le contrôle de la rupture est toujours incomplet. $\mathrm{Ce}$ comportement particulier peut s'expliquer par la structure de la roche. Le grès est très poreux et les inégalités de dilatation thermique des différents grains ne conduisent pas à la formation de fissures, mais à un matériau plus compact qui est, par conséquent, plus résistant.

Si l'on compare le grès des Vosges et le calcaire d'Euville, qui a également une porosité élevée, on constate que la résistance de ce dernier diminue avec 


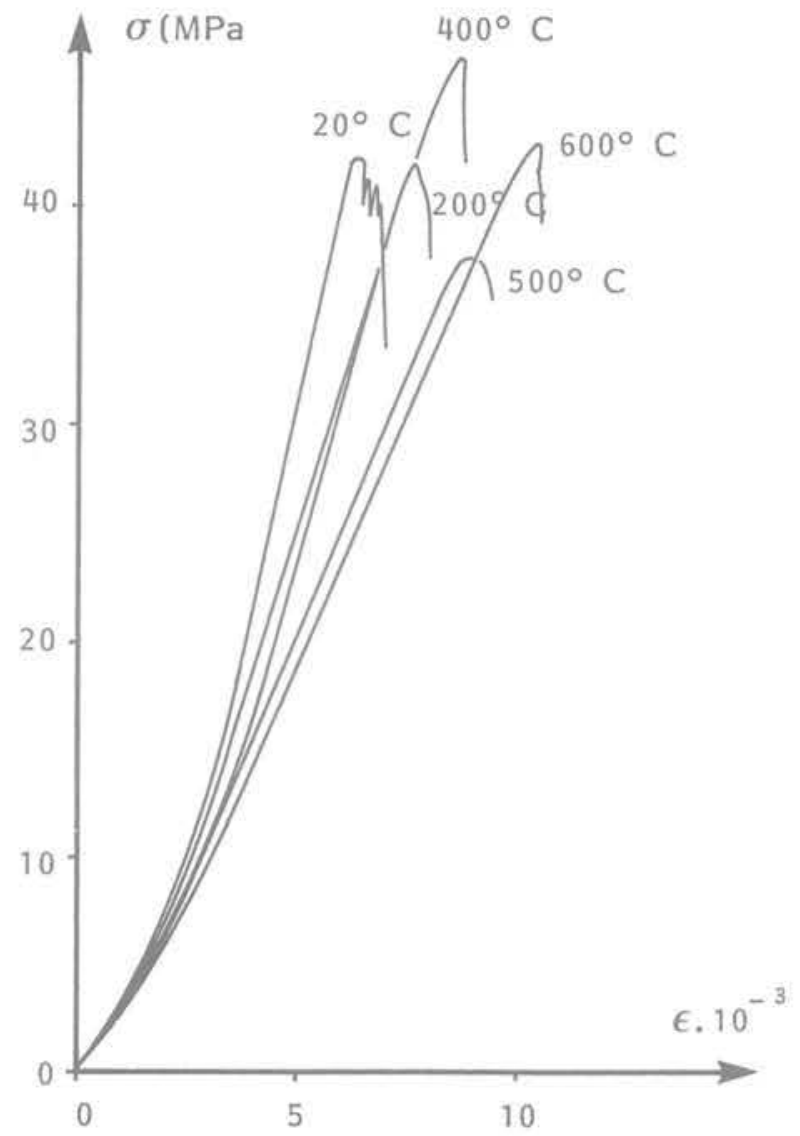

Fig. 5. - Courbes contrainte-déformation du grès des Vosges à différentes températures $\left(\dot{\varepsilon}=10^{-5} s^{-1}\right)$.

la température, dans une proportion toutefois moins importante que dans le cas des roches du type granite. Cependant, deux raisons peuvent être à l'origine de cette différence de comportement entre le grès et le calcaire. D'une part, la calcite est un minéral plus sensible aux variations de température que le quartz; d'autre part, la dimension des grains de calcite est plus élevée que celle des grains de quartz, ce qui entraîne des différences de variations au niveau des dilatations des grains d'une roche à l'autre.

En reprenant les effets conjugués de la variation de la température et de celle de la vitesse de sollicitation, on devrait s'attendre, dans le cas du grès, à une diminution de la résistance ultime avec une augmentation de la vitesse de déformation. Cependant, dans la gamme des vitesses utilisées $\left(5.10^{-7}\right.$ à $\left.10^{-3} \mathrm{~s}^{-1}\right)$, on observe une augmentation de la résistance avec la vitesse de sollicitation.

\section{RELAXATION SOUS TEMPÉRATURE}

La relaxation des contraintes dans un matériau soumis à une charge est une des manifestations de l'influence du temps sur le comportement mécanique. Par relaxa- tion, il faut entendre diminution de la contrainte en fonction du temps, quel que soit l'état du matériau.

Des essais de relaxation ont été effectués à plusieurs niveaux de contrainte pendant un essai de compression et pour différentes températures. Ces niveaux de contrainte ont été définis de la manière suivante (fig. 6): la première relaxation est réalisée avant la

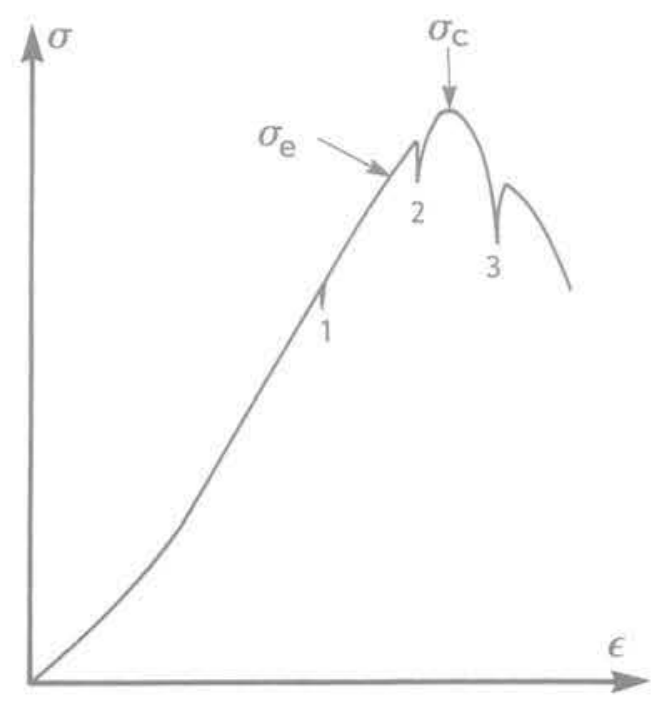

Fig. 6. - Essais de relaxation.

$1,2,3$ niveaux de contrainte de relaxation $\sigma_{0}$

$\left(\sigma_{\theta}=\right.$ limite élastique, $\sigma_{c}=$ résistance ultime $)$.

limite élastique, la deuxième entre la limite élastique et la résistance ultime et la troisième dans la phase post-maximum. La diminution de la contrainte a été enregistrée en fonction du temps. La figure 7 donne un exemple des courbes obtenues pour un niveau de contrainte égal à environ $80 \%$ de la limite élastique, à différentes températures, dans le cas du granite de Senones.

La relaxation des contraintes est une propriété spécifique des matériaux visco-élastiques, pour lesquels la diminution des contraintes est une fonction exponentielle du temps. En vue d'interpréter les résultats expérimentaux, nous les avons comparés à une fonction du même type:

$$
\sigma=\sigma_{0} \exp \left(-\mathrm{t} / \mathrm{t}_{\mathrm{r}}\right) \text {. }
$$

Dans cette relation, $t_{r}$ désigne le temps de relaxation et $\sigma_{0}$ le niveau de contrainte.

Le tableau II donne des valeurs de $t_{\mathrm{s}}$ pour les granites de Senones et de La Clarté, ainsi que pour le marbre de Carrare, en fonction de la température pour différentes valeurs de $\sigma_{0}$. On constate que, pour un niveau de contrainte donné, le temps de relaxation décroît lorsque la température augmente. Des considérations basées sur l'équation d'Arrhénius permettent de relier $t_{r}$ à la température $\mathrm{T}$ au moyen de la relation (FREUDENTHAL, 1950):

$$
t_{\mathrm{r}}=\mathrm{t}_{\mathrm{r} 0} \exp (\mathrm{Q} / \mathrm{RT})
$$




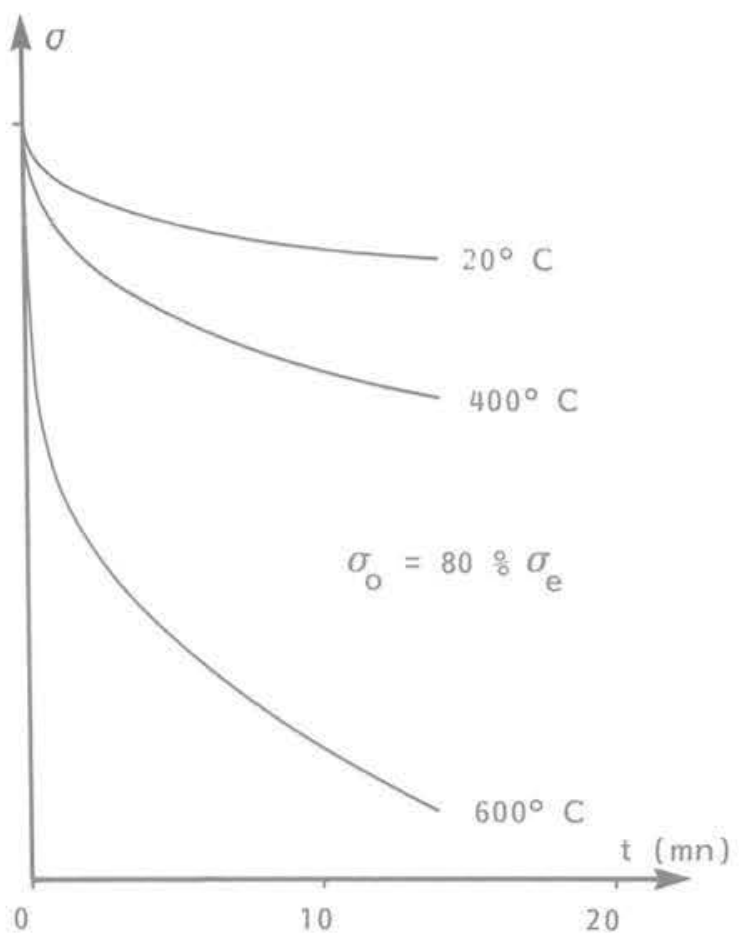

Fig. 7. - Exemple de courbes de relaxation du granite de Senones à différentes températures.

Tableau II. - Temps de relaxation $t_{r}$, en minutes, pour le granite de Senones (GS), le granite de La Clarté (GC) et le marbre de Carrare (MC) à différentes températures et pour différents niveaux de contrainte

\begin{tabular}{|c|c|c|c|c|}
\hline \multirow{2}{*}{\multicolumn{2}{|c|}{$\begin{array}{c}\text { Niveau } \\
\text { de contrainte } \\
\sigma_{\circ}\end{array}$}} & \multicolumn{3}{|c|}{ Temps de relaxation $t_{r}$ (min) } \\
\hline & & $\begin{array}{c}1 \\
\text { inférieur } \\
\text { à } \sigma_{e}\end{array}$ & $\begin{array}{c}2 \\
\text { inférieur } \\
\text { à } \sigma_{c}\end{array}$ & $\begin{array}{c}3 \\
\text { post- } \\
\text { maximum }\end{array}$ \\
\hline GS & $\begin{array}{c}\mathrm{T} \\
400^{\circ} \mathrm{C} \\
600^{\circ} \mathrm{C}\end{array}$ & $\begin{array}{r}250 \\
95 \\
62\end{array}$ & $\begin{array}{r}187 \\
48 \\
30\end{array}$ & $\begin{array}{r}163 \\
30 \\
27\end{array}$ \\
\hline GC & $\begin{array}{c}\mathrm{T} \\
400^{\circ} \mathrm{C} \\
600^{\circ} \mathrm{C}\end{array}$ & $\begin{array}{r}193 \\
37 \\
34\end{array}$ & $\begin{array}{r}186 \\
31 \\
30\end{array}$ & $\begin{array}{l}91 \\
12 \\
20\end{array}$ \\
\hline $\mathrm{MC}$ & $\begin{array}{c}T^{\circ} \\
400^{\circ} \mathrm{C} \\
600^{\circ} \mathrm{C}\end{array}$ & $\begin{array}{r}180 \\
37 \\
9\end{array}$ & $\begin{array}{l}95 \\
28 \\
10\end{array}$ & $\begin{array}{l}30 \\
20 \\
12\end{array}$ \\
\hline
\end{tabular}

$t_{\text {ro }}$ étant une constante ayant la dimension d'un temps. Cette relation exponentielle met en évidence la forte influence d'une variation de température sur le temps de relaxation. Lorsque la température croît, la "viscosité » du matériau diminue et la relaxation des contraintes est plus rapide.
L'ajustement de la relaxation des contraintes à un modèle exponentiel donne lieu à une bonne corrélation pour les essais de relaxation effectués avant la résistance ultime, quelle que soit la température. Au cours de la phase de radoucissement, la corrélation est moins bonne; on peut dire que le comportement du matériau s'éloigne du modèle visco-élastique par suite de l'apparition de phénomènes liés au développement trop important de la fracturation au cours de la relaxation, la rupture localisée donnant lieu à une relaxation instantanée des contraintes.

L'expérience montre d'ailleurs (tableau II) que le temps de relaxation, mesuré en différents points de la courbe $\sigma-\varepsilon$, diminue à mesure que ces points se situent dans des domaines successifs de plus en plus fracturés.

\section{INFLUENCE SIMULTANÉE DE LA TEM- PÉRATURE ET DE LA VITESSE DE SOLLI- CITATION SUR LA RÉSISTANCE ULTIME}

A température ordinaire, la vitesse de sollicitation joue un rôle important sur la valeur de la résistance à la compression, qui suit une loi du type (HOUPERT, 1966)

$$
\sigma_{\mathrm{c}}=\sigma_{0}+\sigma_{1} \log \dot{\varepsilon} / \dot{\varepsilon}_{0}
$$

relation dans laquelle $\dot{\varepsilon}$ désigne la vitesse de déformation, $\sigma_{0}, \sigma_{1}$ et $\varepsilon_{0}$ des constantes. Cette dépendance de $\sigma_{c}$ vis-à-vis de $\dot{\varepsilon}$ résulte de l'influence du temps sur le développement de la microfracturation. Le comportement différé à la rupture des roches est lié à la relaxation des contraintes; celle-ci est d'autant plus rapide que

- la température est élevée,

- le matériau est hétérogène et discontinu,

- la vitesse de sollicitation est faible.

Autrement dit, les effets «visqueux» sont moins prononcés pour les températures élevées, les roches fissurées et hétérogènes et les essais lents. On peut donc penser que, sous température, la variation de vitesse de sollicitation a moins d'influence sur la résistance ultime à température élevée qu'à basse température.

Des essais de compression ont été effectués sur le granite de Senones à $600^{\circ} \mathrm{C}$ pour différentes valeurs de $\dot{\varepsilon}$. Un ajustement à une loi logarithmique est présenté sur la figure 8 , en prenant $\dot{\varepsilon}_{0}=10^{-8} \mathrm{~s}^{-1}$; cette figure donne également la variation de $\sigma_{c}$ pour la roche testée à température ambiante. On peut donner les variations suivantes:

- granite témoin: $\sigma_{\mathrm{c}}=125+9 \log \dot{\varepsilon}$;

- granite à $600{ }^{\circ} \mathrm{C}: \sigma_{\mathrm{c}}=30+3 \log \dot{\varepsilon}$,

On constate effectivement en comparant les pentes des deux droites, que l'influence de la variation de $\dot{\varepsilon}$ sur $\sigma_{c}$ est plus faible aux températures élevées qu'aux basses températures. 


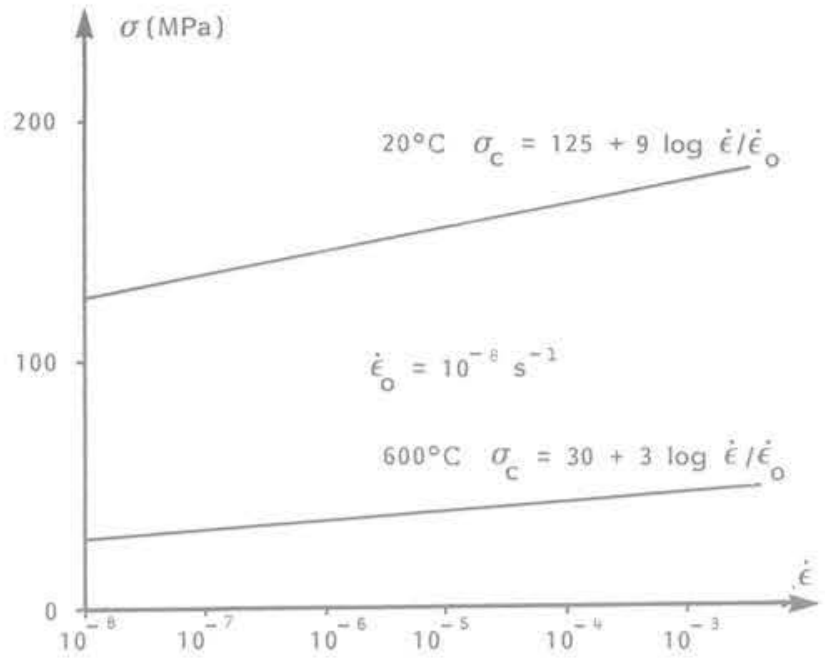

Fig. 8. - Granite de Senones: Augmentation de la résistance en fonction de la vitesse de sollicitation à $20^{\circ} \mathrm{C}$ et $600^{\circ} \mathrm{C}$.

\section{CONCLUSION}

D'une manière générale, une augmentation de température produit une diminution de la résistance ultime et du module de déformation, un accroissement de la phase post-maximum et un changement de pente de la courbe contrainte-déformation dans cette phase. Ces variations sont identiques à celles que l'on observe en fonction de la vitesse de sollicitation (HOUPERT, 1974), dans ce sens qu'une augmentation de la température produit le même effet qu'une diminution de la vitesse de sollicitation. Dans le cas de roches entièrement cristallisées (granites) et des calcaires, ces variations sont monotones; cependant, dans le cas du grès, qui est composé de grains de quartz reliés par un ciment, elles sont plus complexes et encore mal définies.

La relaxation des contraintes dépend de la température. Cette liaison découle de l'équation d'Arrhénius et elle a des conséquences importantes sur le comportement ã la rupture sous température des roches.

Il est possible de comparer le comportement en compression simple des roches sous température à celui des roches préfissurées thermiquement, c'est-àdire ayant été soumises, avant sollicitation mécanique, à une température donnée suivie d'un retour à la température ambiante (HOUPERT et HOMANDÉTIENNE, 1979). Dans le premier cas, les fissures thermiques existent dans le matériau, mais elles restent, zn grande partie, fermées par suite de la dilatation des grains. Dans le second cas, après refroidissement, les fissures sont, pour la plupart, ouvertes. Il y a, bien entendu, de grandes analogies entre les deux comportements. Cependant, le contrôle de la rupture est généralement plus difficile sous température que si la roche est préfissurée.
Dans les roches à quartz, il se produit une fissuration thermique importante au-dessus de $600^{\circ} \mathrm{C}$. Pour des granites préfissurés, on distingue nettement deux groupes de courbes contrainte-déformation: celles relatives à des températures inférieures à $500^{\circ} \mathrm{C}$ et celles relatives à des températures supérieures (HOUPERT et HOMAND-ÉTIENNE, 1979). Cette distinction est surtout importante pour les vitesses de déformation faibles; elle disparaît pour les vitesses de déformation élevées. Par contre, elle n'existe pas pour les roches testées sous température, par suite de la non-ouverture des fissures thermiques dans ce cas.

\section{RÉFÉRENCES BIBLIOGRAPHIQUES}

ABEGA EBOLO, T. (1978). - Etude du développement de la microfracturation thermique par la célérité des ondes et comportement des roches en compression uniaxiale. - D.E.A., I.N.P.L., Nancy.

BRIGHENTI, G. (1970). - Influence of cryogenic temperatures on the mechanical characteristics of rocks. - C.R. $2^{e}$ Congr. Internat. Méc. Roches, Belgrade, vol. 1, comm. 2-27, $5 \mathrm{p}$.

FREUDENTHAL, A.M. (1950). - The inelastic behavior of engineering materials and structures. Wiley, New York, $587 \mathrm{p}$.

HOUPERT, R. (1966). - Variation de la résistance à la compression simple des roches grenues en fonction de la vitesse de mise en charge. - C.R. Acad. Sci., vol. 262, série A, p. 1423-1425.

HOUPERT, R. (1970). - La résistance à la rupture des roches en compression simple. - C.R. $2^{e}$ Congr. Internat. Méc. Roches, Belgrade, vol. 2, comm. 3-8, $7 \mathrm{p}$.

HOUPERT, R. (1974). - Le rôle du temps dans le comportement à la rupture des roches. - C.R. $3^{e}$ Congr. Internat. Méc. Roches, Denver, vol. 2 , t. A, p. $325-329$.

HOUPERT, R. (1979). - Le comportement à la rupture des roches. - C.R. $4^{e}$ Congr. Internat. Méc. Roches, Montreux, conf. th. 1, vol. 3, p. 115-122.

HOUPERT, R.; HOMAND-ETIENNE, F. (1979). Influence de la température sur le comportement mécanique des roches. - C.R. $4^{e}$ Congr. Internat. Méc. Roches, Montreux, p. 177-180.

KUMAR, A. (1968). - The effect of stress rate and temperature on the strength of basalt and granite. - Geophysics, vol. 33, p. 501-510.

RICHARDS, C. (1965). - La science des matériaux de lingénieur. - Dunod, Paris, $543 \mathrm{p}$.

STEPHANSSON, O.; SWAN, G.; LEIJON, B. (1978). - Mechanical properties of Stripa granite. In situ heating experiments in geological formations. Ludvika-Stripa, 13-15 septembre 1978, p. 191-204.

WINGQUIST, C.F. (1969). - Elastic moduli of rock at elevated temperatures. - U.S. Dep. Inter. Bur. Mines rep. invest. $7269,18 \mathrm{p}$. 
